Analysis and Algebra on Differentiable Manifolds 
P.M. Gadea • J. Muñoz Masqué

\section{Analysis and Algebra on Differentiable Manifolds}

A Workbook for Students and Teachers

舅 Springer 
Prof. P.M. Gadea

Institute of Fundamental Physics

CSIC

Serrano 113-bis

28006 Madrid

Spain

pmgadea@iec.csic.es
Prof. J. Muñoz Masqué

CSIC Madrid

Inst. Física Aplicada

C/ Serrano 144

28006 Madrid

Spain

jaime@iec.csic.es

ISBN 978-90-481-3563-9

e-ISBN 978-90-481-3564-6

DOI 10.1007/978-90-481-3564-6

Springer Dordrecht Heidelberg London New York

Library of Congress Control Number: 2009941371

Mathematics Subject Classification (2000): 0001, 00A07, 00A22, 2201, 5301, 53B21, 55R10, 5701, 5801, $58 \mathrm{~A} 10,58 \mathrm{C} 35$

1st edition: (C) Kluwer Academic Publishers 2001

Revised edition: (C) Springer Science+Business Media B.V. 2009

(C) Springer Science+Business Media B.V. 2001, First softcover printing 2009

No part of this work may be reproduced, stored in a retrieval system, or transmitted in any form or by any means, electronic, mechanical, photocopying, microfilming, recording or otherwise, without written permission from the Publisher, with the exception of any material supplied specifically for the purpose of being entered and executed on a computer system, for exclusive use by the pur-chaser of the work.

Cover design: WMXDesign GmbH, Heidelberg

Printed on acid-free paper

Springer is part of Springer Science+Business Media (www.springer.com) 
To Mary 


\section{Foreword}

A famous Swiss professor gave a student's course in Basel on Riemann surfaces. After a couple of lectures, a student asked him, "Professor, you have as yet not given an exact definition of a Riemann surface." The professor answered, "With Riemann surfaces, the main thing is to UNDERSTAND them, not to define them."

The student's objection was reasonable. From a formal viewpoint, it is of course necessary to start as soon as possible with strict definitions, but the professor's answer also has a substantial background. The pure definition of a Riemann surfaceas a complex 1-dimensional complex analytic manifold-contributes little to a true understanding. It takes a long time to really be familiar with what a Riemann surface is.

This example is typical for the objects of global analysis-manifolds with structures. There are complex concrete definitions but these do not automatically explain what they really are, what we can do with them, which operations they really admit, how rigid they are. Hence, there arises the natural question-how to attain a deeper understanding?

One well-known way to gain an understanding is through underpinning the definitions, theorems and constructions with hierarchies of examples, counterexamples and exercises. Their choice, construction and logical order is for any teacher in global analysis an interesting, important and fun creating task.

This workbook, using a very cleverly composed series of exercises and examples covering the whole area of manifolds, Lie groups, fibre bundles and Riemannian geometry, will enable the reader a deeper understanding and feeling for Riemann surfaces.

Jürgen Eichhorn Greifswald University 


\section{Preface}

This book is intended to cover the exercises of standard courses on analysis and algebra on differentiable manifolds for advanced undergraduate and graduate years, with specific focus on Lie groups, fibre bundles and Riemannian geometry. It will prove of interest for students in mathematics and theoretical physics, and in some branches of engineering.

It is not intended as a handbook on those topics, in the form of problems, but merely as a practical complement to the courses, often found in excellent books, as cited in the bibliography.

The prerequisites are linear and multilinear algebra, calculus on several variables and various concepts of point-set topology.

The first six chapters contain 375 solved problems sorted according to the aforementioned topics. These problems fall, "grosso modo," into four classes:

(1) Those consisting of mere calculations, mostly elementary, aiming at checking a number of notions on the subjects.

(2) Problems dedicated to checking some specific properties introduced in the development of the theory.

(3) A class of somewhat more difficult problems devoted to focusing the attention on some particular topics.

(4) A few problems introducing the reader to certain questions not usually explained. The level of these problems is quite varied, ranging from those handling simple properties to others that need sophisticated tools.

Throughout the book, differentiable manifolds, functions, and tensors fields are assumed to be of class $C^{\infty}$, mainly to simplify the exposition. We call them, indiscriminately, either differentiable or $C^{\infty}$.

Similarly, manifolds are supposed to be Hausdorff and second countable, though a section is included analysing what happens when these properties fail, aimed at a better understanding of the meaning of such properties.

The Einstein summation convention is used.

Chapter 7 provides a selection of the theorems and definitions used throughout the book, but restricted to those whose terminology could be misleading for the lack of universal acceptance. Moreover, to solve some types of problems, certain 
definitions and notations should be precisely fixed; recalling the exact statement of some theorems is often convenient in practice as well. However, this chapter has by no means the intention of being either a development or a digest of the theory.

Chapter 8 offers a collection of formulae and tables concerning spaces and groups frequent in differential geometry. Many of them are used throughout the book; others are not, but they have been included since such a collection should be useful as an aide-mémoire, even for teachers and researchers. As in Chapter 7, no effort to be exhaustive has been attempted.

We hope the book will render a good service to teachers and students of differential geometry and related topics. While no reasonable effort has been spared to ensure accuracy and precision, the attempt of writing such a book necessarily will contain misprints, and probably some errors. Any corrections, suggestions or comments helping to improve future editions will be highly appreciated. The reader is kindly requested to send his/her opinions to pmgadea@iec.csic.es or jaime@ iec.csic.es

In this corrected reprint we have corrected a couple of dozens of typos, slightly modified the statement of Problem 1.1.13, and changed the proof of Problem 5.3.6, (2).

Madrid, August 2009

The authors 


\section{Acknowledgements}

Our hearty thanks to the referees and to Professors Marco Castrillón López, Raúl Durán Díaz, Alberto Elduque, Fernando Etayo, Olga Gil-Medrano, Carolyn Gordon, Angel Montesinos Amilibia, José A. Oubiña, Encarna Reyes and Gustavo Santos García for their valuable help, corrections, and suggestions.

We are indebted to Arturo Quijada Arteaga for his excellent work in the graphics.

Our special thanks to Professor Jürgen Eichhorn, who kindly accepted our invitation to write the Foreword. 


\section{Contents}

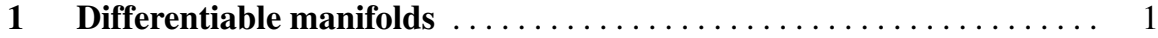

$1.1 \quad C^{\infty}$ manifolds . . . . . . . . . . . . . . . . . . . . . . . 1

1.2 Differentiable Structures Defined on Sets ............... 13

1.3 Differentiable Functions and Mappings ................ 21

1.4 Critical Points and Values......................... 28

1.5 Immersions, Submanifolds, Embeddings and Diffeomorphisms ... 31

1.6 Constructing Manifolds by Inverse Image. Implicit Map Theorem .. 40

1.7 Submersions. Quotient Manifolds ..................... 45

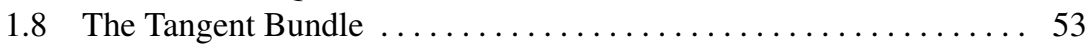

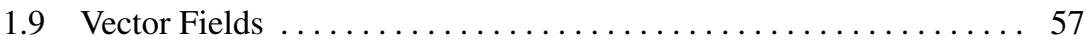

1.9.1 Working with Vector Fields ................... 57

1.9 .2 Integral Curves . . . . . . . . . . . . . . . . . . . . . . . 64

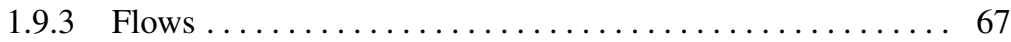

1.9.4 Transforming Vector Fields $\ldots \ldots \ldots \ldots \ldots \ldots \ldots \ldots \ldots$

2 Tensor Fields and Differential Forms $\ldots \ldots \ldots \ldots \ldots \ldots \ldots \ldots \ldots \ldots$

$2.1 \quad$ Vector Bundles................................. 75

2.2 Tensor and Exterior Algebras. Tensor Fields . . . . . . . . . . 82

2.3 Differential Forms. Exterior Product ..................... 84

2.4 Lie Derivative. Interior Product $\ldots \ldots \ldots \ldots \ldots \ldots \ldots \ldots \ldots \ldots \ldots \ldots \ldots \ldots$

2.5 Distributions and Integral Manifolds. Frobenius' Theorem.

Differential Ideals................................ 97

2.6 Almost Symplectic Manifolds .......................... 104

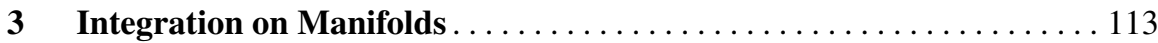

3.1 Orientable manifolds. Orientation-preserving maps $\ldots \ldots \ldots \ldots 113$

3.2 Integration on Chains. Stokes' Theorem I . . . . . . . . . . . 116

3.3 Integration on Oriented Manifolds. Stokes' Theorem II . . . . . . . . . . 119

3.4 De Rham Cohomology .......................... 123 


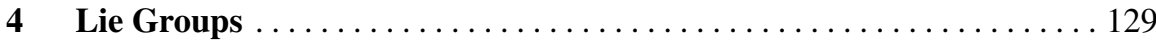

4.1 Lie Groups and Lie Algebras ........................... 129

4.2 Homomorphisms of Lie Groups and Lie Algebras ............ 141

4.3 Lie Subgroups and Lie Subalgebras .................... 146

4.4 The Exponential Map . . . . . . . . . . . . . . . . . . . . . . . . . . . 154

4.5 The Adjoint Representation .......................... 160

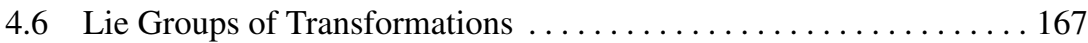

4.7 Homogeneous Spaces ............................... 174

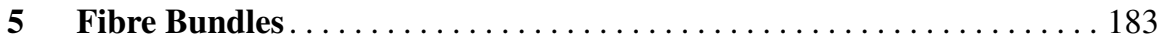

5.1 Principal Bundles ............................... 183

5.2 Connections in Bundles ............................. 192

5.3 Characteristic Classes ................................... 197

5.4 Linear Connections . . . . . . . . . . . . . . . . . . . . . . . . . 211

5.5 Torsion and Curvature .............................. 213

5.6 Geodesics .................................... 220

5.7 Almost Complex Manifolds ........................... 224

6 Riemannian Geometry ............................... 233

6.1 Riemannian Manifolds .............................. 233

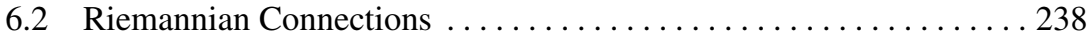

6.3 Geodesics ..................................... 243

6.4 The Exponential Map ............................. 248

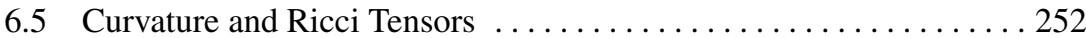

6.6 Characteristic Classes . . . . . . . . . . . . . . . . . . . . . . . . . . 256

6.7 Isometries . . . . . . . . . . . . . . . . . . . . . . . . 260

6.8 Homogeneous Riemannian Manifolds and Riemannian Symmetric Spaces................................... 267

6.9 Spaces of Constant Curvature ..................... 276

6.10 Left-invariant Metrics on Lie Groups ................... 279

6.11 Gradient, Divergence, Codifferential, Curl, Laplacian, and Hodge

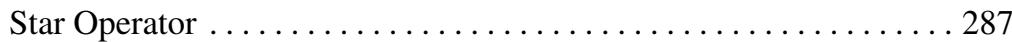

6.12 Affine, Killing, Conformal, Projective, Jacobi, and Harmonic Vector Fields . . . . . . . . . . . . . . . . . . . . . . . . . . . . 299

6.13 Submanifolds. Second Fundamental Form . . . . . . . . . . . . . . . 313

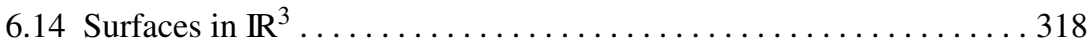

6.15 Pseudo-Riemannian Manifolds ......................... 325

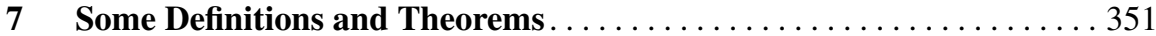

7.1 Chapter 1. Differentiable Manifolds ..................... 351

7.2 Chapter 2. Tensor Fields. Differential Forms ............... 356

7.3 Chapter 3. Integration on Manifolds ..................... 360

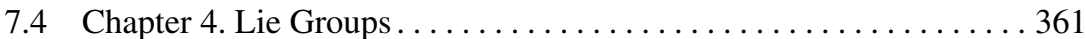

7.5 Chapter 5. Fibre Bundles ......................... 366

7.6 Chapter 6. Riemannian Geometry ...................... 370 
8 Some Formulas and Tables $\ldots \ldots \ldots \ldots \ldots \ldots \ldots \ldots \ldots$

References ..................................419

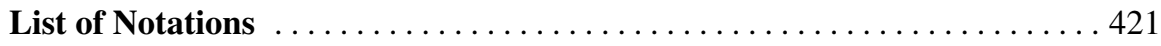

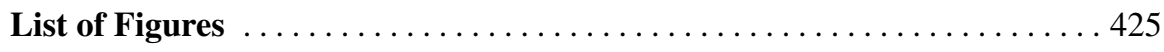

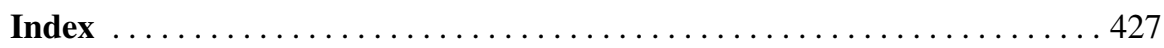

